\title{
A associação entre fatores psicossociais e atividade física em adolescentes é moderada por fatores sociodemográficos?
}

\author{
The association between psychosocial factors and physical activity in \\ adolescents is moderated by demographic factors?
}

Luanna Alexandra Cheng ${ }^{1,2}$, Gerfeson Mendonça1,2, José Cazuza de Farias Júnior $r^{1,2,3}$

\section{Resumo}

O objetivo deste estudo foi analisar se a associação entre os fatores psicossociais e o nível de atividade física é moderada pelos fatores sociodemográficos em adolescentes. Utilizou-se dados completos de 2.540 adolescentes (57\% do sexo feminino, média de idade $=16,4 ; d p=1,2$ anos), de escolas públicas e privadas de ensino médio no município de João Pessoa (PB). A atividade física foi mensurada por questionário. Os fatores psicossociais mensurados foram: percepção de autoeficácia e o apoio social dos pais e amigos para prática de atividade física. Os resultados da análise por regressão linear múltipla demostraram que o sexo moderou a associação da percepção de autoeficácia (masculino $\beta=19,9$ vs feminino $\beta=9,6$ ), apoio social dos pais (masculino $\beta=59,9$ vs feminino $\beta=35,1$ ) e dos amigos (masculino $\beta=68,3$ vs feminino $\beta=40,1$ ) com o nível de atividade física. A classe econômica moderou a associação do apoio social dos pais $(A / B \beta=38,2$ vs $C / D / E \beta=54,4)$ e dos amigos (A/B $\beta=53,1$ vs $\mathrm{C} / \mathrm{D} / \mathrm{E} \beta=42,3)$ com o nível de atividade física, e a idade (1415 anos $\beta=54,2$ vs 16-19 anos $\beta=43,6)$ moderou a associação do apoio social dos amigos com o nível de atividade física. Concluise que as possíveis influências da percepção de autoeficácia e do apoio social sobre os níveis de prática de atividade variam conforme as características sociodemográficas dos adolescentes.

\section{Palavras-chave}

Apoio social; Autoeficácia; Atividade motora; Moderadores; Adolescência.

\begin{abstract}
The objective this study was analyze whether the association between psychosocial factors and physical activity is moderated by sociodemographic factors in adolescents. This study used complete data from 2.540 adolescents (57\% female, mean age $=16.4$, $s d=1.2$ years), from public and private high schools in the city of Joao Pessoa (PB). Physical activity was measured by questionnaire. Psychosocial factors measured was: perception of self-efficacy and social support to physical activity from parents and friends. The results of the multiple linear regression demonstrated that sex moderated the association between self-efficacy ( $\beta=19.9$ male vs female $\beta=9.6)$, social support from parents (male $\beta=59.9$ v female $\beta=35.1$ ) and friends (male $\beta=68.3$ vs female $\beta=40.1$ ) and physical activity. The economy status moderated the association between social support from parents $(A / B \beta=38.2$ vs $C / D / E \beta=54.4)$ and physical activity. Age (14-15 years $\beta=54.2$ vs $16-19$ years $\beta=43.6)$ and economic status $(A / B \beta=53.1$ vs $C / D / E \beta=42.3)$ were a moderator of the association between social support from friends and physical activity. It is concluded that the possible influences of perceived self -efficacy and social support on the physical activity levels may vary according to adolescent's sociodemographic characteristics.
\end{abstract}

\section{Keywords}

Social support; Self-efficacy; Motor activity; Moderators; Adolescence.

\section{Introdução}

Na última década, observou-se um aumento no número de estudos que tinham como foco a identificação dos fatores que podem influenciar na participação dos adolescentes em atividades físicas e os mecanismos pelos

1 Programa Associado de Pós-Graduaçăo em Educação Física UPE/UFPB, João Pessoa, Brasil.

2 Grupo de Estudos e Pesquisas em Epidemiologia da Atividade Física - GEPEAF, João Pessoa, Brasil.

3 Universidade Federal da Paraíba - UFPB, João Pessoa, Brasil. quais esses fatores atuam sobre esse comportamento ${ }^{1-3}$.

Essa preocupação se deve ao fato de que as prevalências de adolescentes que não atingem as recomendações mínimas de prática de atividade física têm se mostrado elevadas ${ }^{4,5}$, há uma redução no nível de atividade física durante a adolescência ${ }^{6}$, a prática de atividade física nesta fase da vida pode ser transferida à idade adulta e os níveis de prática podem influenciar de forma mediata e imediata na saúde dos adolescentes ${ }^{7,8}$. 
Nesse sentido, tem sido recomendado o desenvolvimento de ações para aumentar a participação dos adolescentes em atividades físicas ${ }^{3}$. Para tanto, observase a necessidade de se identificar e intervir sobre os fatores correlatos e os determinantes da atividade física. A atividade física tem se mostrado associada a fatores intrapessoais, interpessoais e ambientais ${ }^{2,3}$. Dentre os fatores intra e interpessoais, a autoeficácia e o apoio social para a prática de atividade física, respectivamente, são os fatores psicossociais mais investigados ${ }^{1,9}$. Estes fatores são passiveis de serem modificados por meio de intervenções e estão consistentemente associados a maiores níveis de atividade física em adolescentes ${ }^{1,9}$. Porém, pouco se sabe se os fatores psicossociais influenciam de maneira diferente a prática de atividade física conforme as características sociodemográficos dos adolescentes (sexo, idade e condição socioeconômica) ${ }^{10}$.

Os estudos têm apontado que os adolescentes do sexo feminino, os mais velhos e aqueles de condições socioeconômicas mais baixas apresentam menores níveis de atividade física ${ }^{4,8}$. Sendo assim, é possível que a magnitude e/ou o sentido da medida de associação entre a percepção de autoeficácia, apoio social e o nível de atividade física sejam distintos entre adolescentes de diferentes sexos, idades e classes econômicas, resultando num possível efeito moderador dos fatores sociodemográficos para essas associações.

Uma variável moderadora é aquela que modifica o sentido ou a magnitude da medida de associação entre uma variável independente e a dependente, sendo essa associação diferente em uma ou mais categorias dessa terceira variável ${ }^{11}$. Estudos que analisaram a associação entre diferentes fatores interpessoais, intrapessoais e ambientais, e a prática de atividade física demonstram que as magnitudes das associações foram mais fortes em alguns subgrupos (adolescentes do sexo masculino, mais novos e que residiam em bairros ricos com melhor infraestrutura para prática $)^{1,9}$. Entretanto, pouco se sabe sobre o possível papel moderador das variáveis sociodemográficas na associação da atividade física com a percepção de autoeficácia e do apoio social da família e dos amigos nesse grupo populacional.

Analisar se a percepção de autoeficácia e o apoio social estão associados de maneira diferente com a atividade física segundo o sexo, idade e classe econômica dos adolescentes poderão ajudar a esclarecer o papel que essas variáveis podem ter na determinação dos níveis de atividade física, bem como a direcionar as ações de intervenção sobre atividade física quando essas variáveis forem o alvo das mesmas. Desse modo, este estudo teve por objetivo analisar se a associação entre os fatores psicossociais e o nível de atividade física é moderada pelos fatores sociodemográficos em adolescentes.

\section{Métodos}

Este estudo epidemiológico transversal analisou dados de uma pesquisa intitulada "Nivel de atividade física e fatores associados em adolescentes do ensino médio na cidade de João Pessoa - PB: uma abordagem ecológica", realizada em 2009, com escolares do ensino médio, de 14 a 19 anos de idade, da rede pública e privada de ensino médio do município de João Pessoa (PB).

Para determinação do tamanho da amostra foi adotada uma prevalência estimada de 50\% (300 minutos ou mais por semana de atividade física moderada a vigorosa); erro máximo aceitável de três pontos percentuais; intervalo de confiança de 95\%; efeito de desenho (deff) igual a dois e acréscimo de $30 \%$ para perdas e recusas, resultando em uma amostra de 2.686 adolescentes.

A seleção da amostra foi realizada por conglomerado em dois estágios. No pri- 
meiro, foram selecionadas sistematicamente 30 escolas de ensino médio dentre as 82 (44 públicas e 38 privadas) consideradas elegíveis (escolas que tinha pelo menos uma turma por série de ensino médio), com probabilidade proporcional ao tamanho da mesma (número de alunos regularmente matriculados), distribuídas proporcionalmente por tipo (públicas e privadas) e regiões do município (norte, sul, leste, oeste). No segundo, foram selecionadas 135 turmas, distribuídas proporcionalmente por turno (diurno e noturno) e série de ensino médio $\left(1^{\mathrm{a}}, 2^{\mathrm{a}}\right.$ e $3^{\mathrm{a}}$ séries).

A coleta de dados foi realizada no período de maio a setembro de 2009, por equipe previamente treinada e que participou de estudo piloto, sendo composta por seis alunos do curso de Educação Física. Todas as informações foram coletadas por um questionário validado ${ }^{12,13}$, preenchido pelos adolescentes, na sala de aula, em horário regular, seguindo instruções da equipe de coleta.

As variáveis sociodemográficas analisadas foram: sexo (masculino e feminino); idade (categorizada em: 14-15 e 16-19 anos de idade) e classe econômica (metodologia da Associação Brasileira de Empresas de Pesquisa - ABEP $)^{14}$ que leva em consideração a presença de bens materiais, o número de empregados mensalistas na residência e escolaridade do chefe de família. As pessoas foram agrupadas nas classes econômicas A1, A2, B1, B2, C1, C2, D e E, e reagrupadas nas classes A/B (classe econômica alta) e C/D/E (classe econômica média-baixa). O índice de massa corporal $\left(\mathrm{IMC}=\right.$ massa corporal $[\mathrm{kg}] /$ estatura $\left.[\mathrm{m}]^{2}\right)$ foi determinado com base nas medidas autorreferidas de massa corporal $(\mathrm{kg})$ e estatura $(\mathrm{m})$.

As medidas de todas as variáveis sociodemográficas apresentaram níveis satisfatórios de reprodutibilidade, com valores de kappa iguais ou superiores a 0,89. O IMC apresentou coeficiente de correlação intraclasse (CCI) igual a 0,95 (amostra com 248 adolescentes que não fizeram parte do estudo principal, $57 \%$ do sexo feminino, com média de idade igual a 16,4 anos e desvio padrão igual a 1,2).

A atividade física foi mesurada por questionário ${ }^{12}$. Os adolescentes informaram a frequência (dias/semana) e a duração (minutos/dia) das atividades físicas de intensidade moderada a vigorosa e o deslocamento ativo (caminhada e/ou bicicleta), praticadas por pelo menos 10 minutos, na semana anterior a coleta de dados. Determinou-se o nível de atividade física somando o produto do tempo pela frequência de prática em cada atividade, resultando num escore em minutos por semana.

A percepção de autoeficácia para a prática de atividade física foi mensurada por uma escala composto por dez itens (consistência interna mensurada pelo alpha de Cronbach $(\alpha)=0,76$ e reprodutibilidade: $\mathrm{CCI}=0,75$, IC95\%: $0,58-0,75)^{13}$ que consideraram o quanto os adolescentes se percebiam capazes de praticar atividade física na presença de barreiras. Os itens foram ancorados por escala Likert de quatro pontos (discordo muito $=1$, discordo $=2$, $\operatorname{con} \operatorname{cordo}=3$, concordo muito $=4$ ). Foram criados escores de autoeficácia com base no somatório dos valores atribuídos a cada resposta, podendo variar de 10 a 40 pontos.

O apoio social dos pais e dos amigos para a prática de atividade física foi mensurado por escala com 10 itens, cinco itens para cada (apoio social dos pais $-\alpha=0,81 \mathrm{e}$ CCI $=0,91$ [IC95\%: 0,88-0,93]; apoio social dos amigos $-\alpha=0,90$ e CCI $=0,89$ [IC95\%: $0,87-0,92]^{13}$ ). Os adolescentes informaram a frequência (nunca $=1$, raramente $=2$, frequentemente $=3$, sempre $=4$ ) com que os pais e os amigos ofereciam algum tipo de apoio social para a prática de atividade física (estimular, praticar junto, assistir, comentar, convidar e transportar) durante uma semana típica ou normal. Com base no somatório dos valores atribuídos a cada item foram criados escores que variaram de 5 a 20 pontos. Para os escores de autoeficácia e de apoio social, os maiores valores indicavam níveis mais elevados para essas variáveis. 
Para a análise descritiva foi realizada a distribuição de frequência para as variáveis categóricas e média, desvio padrão e mediana para as variáveis continuas. A regressão linear múltipla foi utilizada para verificar a associação entre os fatores psicossociais (variáveis independentes) e o nível de atividade física (variável dependente). Para avaliar se os fatores sociodemográficos moderavam a associação entre essas variáveis, foram introduzidos termos de interação nos modelos de análises (multiplicação da possível variável moderadora com a variável independente exemplo: sexo*autoeficácia). No primeiro modelo, as variáveis independentes foram: sexo, autoeficácia e o termo de interação sexo*autoeficácia; no segundo: idade, autoeficácia e o termo de interação idade*autoeficácia; no terceiro modelo: classe econômica, autoeficácia e o termo de interação classe econômica*autoeficácia. Essa mesma sequência de análise foi adotada para o apoio social dos pais e dos amigos.

Em todos os modelos, as variáveis sociodemográficas que não estavam sendo testadas como moderadoras e o estado nutricional foram consideradas potenciais fatores de confusão. Quando o termo de interação foi significativo $(\mathrm{p}<0,05)$ a variável sociodemográfica era classificada como moderadora da associação entre o fator psicossocial em análise e o nível de atividade física. Na sequência, foram realizadas análises estratificadas por essa variável, permitindo avaliar o sentido e a magnitude da medida de associação entre o fator psicossocial e o nível de atividade física dos adolescentes para cada categoria dessa variável. Todas as análises estatísticas foram realizadas no Stata 11.0. O nível de significância adotado foi < 5\%.

O estudo foi aprovado pelo Comitê de Ética em Pesquisa com Seres Humanos do Centro de Ciências da Saúde da Universidade Federal da Paraíba - Protocolo: 0062/2009. Todos os adolescentes menores de 18 anos de idade receberam autorização dos pais ou responsáveis e aqueles com 18 ou mais anos de idade assinaram o termo de consentimento livre e esclarecido para participar deste estudo.

\section{Resultados}

Foram entrevistados 2.859 adolescentes (perdas e recusas totalizaram 17,8\% dos casos), sendo excluídos 319 por não apresentarem dados completos para uma ou mais das variáveis psicossociais analisadas. Não foram observadas diferenças significativas $(\mathrm{p}<0,05)$ entre os adolescentes incluídos e os excluídos nas análises nas variáveis sociodemográficas, psicossociais e nível de atividade física. Dos 2.540 adolescentes com dados completos, a maioria era do sexo feminino (57\%) e na faixa etária de 16-19 anos de idade (60,7\%, média de idade=16,4; dp=1,2). Outras características sociodemográficas, nível de atividade física, fatores psicossociais e o estado nutricional dos adolescentes estão apresentados na tabela 1 .

A tabela 2 apresenta os resultados das análises de moderação para as associações entre os fatores psicossociais e a prática de atividade física dos adolescentes. $\mathrm{O}$ sexo do adolescente moderou a associação da percepção de autoeficácia $(\mathrm{p}<0,015)$, apoio social dos pais $(p<0,001)$ e dos amigos $(p<0,001)$ com o nível de atividade física. A idade moderou a associação do apoio social dos amigos $(p<0,026)$ com o nível de atividade física, e a classe econômica moderou a associação do apoio social dos pais $(\mathrm{p}<0,048)$ e dos amigos $(\mathrm{p}<0,027)$ com o nível de atividade física dos adolescentes.

Observou-se que as magnitudes das medidas de associação dos fatores psicossociais com o nível de atividade física foram superiores nos adolescentes do sexo masculino (autoeficácia - masculino $\beta=19,9$ vs feminino - $\beta=9,6$; apoio social dos pais - masculino $-\beta=59,9$ vs feminino $-\beta=35,1$; e apoio social dos amigos - masculino - $\beta=68,3$ vs feminino $-\beta=40,1)$. A magnitude de associação entre o apoio 
TABELA 1 - Características sociodemográficas, nível de atividade física, autoeficácia, apoio social dos pais e dos amigos e índice de massa corporal em adolescentes no município de João Pessoa (PB), 2009.

\begin{tabular}{lcc}
\hline Variáveis & $n$ & Total \\
\hline \multicolumn{1}{l}{ Sexo } & & \\
\hline Masculino & 1.171 & 42,5 \\
\hline Feminino & 1.581 & 57,5 \\
\hline Idade & & \\
\hline $14-15$ anos & 1.087 & 39,3 \\
\hline $16-19$ anos & 1.685 & 60,7 \\
\hline Classe econômica & & \\
\hline A e B (alta) & 1.132 & 46,1 \\
\hline C, D e E (média-baixa) & 1.323 & M (DP) \\
\hline & Md & $544,3(701,5)$ \\
\hline Nível de atividade física (min/sem) & 301 & $27,5(6,6)$ \\
\hline Autoeficácia (pontos) & 27 & $9,8(3,5)$ \\
\hline Apoio social dos pais (pontos) & 9 & $12,2(4,3)$ \\
\hline Apoio social dos amigos (pontos) & 12 & $20,9(3,3)$ \\
\hline IMC (kg/m²) & 20,4 & \\
\hline
\end{tabular}

Md: mediana; M: média; DP: desvio-padrão; IMC: índice de massa corporal.

TABELA 2 - Análise de moderação entre as variáveis sociodemográficas (sexo, idade e classe econômica) para a associação entre autoeficácia, apoio social dos pais e dos amigos e a prática de atividade física dos adolescentes, João Pessoa (PB), 2009.

\begin{tabular}{|c|c|c|c|c|}
\hline & Variáveis & $\beta$ & Erro padrão & Valor $p$ \\
\hline \multirow[t]{12}{*}{$\mathrm{SexO}^{*}$} & Autoeficácia & & & \\
\hline & Sexo & 156,15 & 121,61 & 0,199 \\
\hline & Autoeficácia & 9,41 & 2,74 & 0,001 \\
\hline & Sexo*Autoeficácia & 10,40 & 4,25 & 0,015 \\
\hline & Apoio social dos pais & & & \\
\hline & Sexo & 141,83 & 81,74 & 0,083 \\
\hline & Apoio social dos pais & 35,24 & 5,12 & 0,001 \\
\hline & Sexo*Apoio social dos pais & 27,69 & 7,67 & 0,001 \\
\hline & Apoio social dos amigos & & & \\
\hline & Sexo & $-176,46$ & 84,45 & 0,037 \\
\hline & Apoio social dos amigos & 31,85 & 4,22 & 0,001 \\
\hline & Sexo*Apoio social dos amigos & 38,81 & 6,33 & 0,001 \\
\hline \multirow[t]{12}{*}{ Idade** } & Autoeficácia & & & \\
\hline & Idade & 56,89 & 122,33 & 0,642 \\
\hline & Autoeficácia & 13,41 & 2,71 & 0,000 \\
\hline & Idade*Autoeficácia & 0,78 & 4,28 & 0,855 \\
\hline & Apoio social dos pais & & & \\
\hline & Idade & 65,19 & 82,59 & 0,430 \\
\hline & Apoio social dos pais & 48,2 & 5,06 & 0,001 \\
\hline & Idade*Apoio social dos pais & $-2,93$ & 7,73 & 0,704 \\
\hline & Apoio social dos amigos & & & \\
\hline & Idade & $-111,18$ & 79,94 & 0,164 \\
\hline & Apoio social dos amigos & 43,77 & 3,98 & 0,001 \\
\hline & Idade*Apoio social dos amigos & 13,72 & 6,15 & 0,026 \\
\hline \multirow{5}{*}{$\begin{array}{l}\text { Classe } \\
\text { econômica*** }\end{array}$} & Autoeficácia & & & \\
\hline & Classe econômica & $-57,11$ & 120,47 & 0,636 \\
\hline & Autoeficácia & 12,42 & 2,89 & 0,000 \\
\hline & Classe econômica*Autoeficácia & 2,76 & 4,22 & 0,512 \\
\hline & Apoio social dos pais & & & \\
\hline
\end{tabular}




\begin{tabular}{lccc}
\hline Variáveis & $\beta$ & Erro padrão & Valor p \\
\hline Classe econômica & 113,04 & 82,36 & 0,170 \\
\hline Apoio social dos pais & 55,04 & 5,63 & 0,000 \\
\hline Classe econômica*Apoio social dos pais & $-15,45$ & 7,81 & 0,048 \\
\hline Apoio social dos amigos & & & \\
\hline Classe econômica & $-135,25$ & 78,19 & 0,084 \\
\hline Apoio social dos amigos & 43,16 & 4,16 & 0,000 \\
\hline Classe econômica*Apoio social dos amigos & 13,31 & 6,02 & 0,027 \\
\hline
\end{tabular}

Grupos de referência: sexo feminino; idade de 16-19 anos; classe média-baixa (classe C, D e E). *Ajustado por idade, classe econômica e índice de massa corporal. **Ajustado por sexo, classe econômica e índice de massa corporal. ${ }^{* *}$ Ajustado por sexo, idade e índice de massa corporal.

social dos pais e o nível de atividade física dos adolescentes foram superiores para os adolescentes de classe econômica média-baixa (A/B - $\beta=38,2$ vs $C / D / E-\beta=54,4)$. As maiores magnitudes de associação entre o apoio social dos amigos e o nível de atividade física dos adolescentes foi superior nos de classe econômica alta (A/B $\beta=53,1$ vs $C / D / E-\beta=42,3)$ e nos mais novos (14-15 anos $-\beta=54,2$ vs $16-19$ anos de idade $-\beta=43,6)$. Todos os resultados apresentaram valor $p<0,001$ - Tabela 3 .

TABELA 3 - Magnitudes das associações entre fatores psicossociais e prática de atividade física em adolescentes, João Pessoa (PB), 2009.

\begin{tabular}{|c|c|c|c|c|c|c|c|c|c|}
\hline \multirow[b]{2}{*}{ Variáveis } & \multicolumn{3}{|c|}{ Autoeficácia } & \multicolumn{3}{|c|}{ Apoio social dos pais } & \multicolumn{3}{|c|}{ Apoio social dos amigos } \\
\hline & $\beta$ & $\begin{array}{c}\beta \\
\text { padronizado }\end{array}$ & $\begin{array}{l}\text { Erro } \\
\text { padrão }\end{array}$ & $\beta$ & $\begin{array}{c}\beta \\
\text { padronizado }\end{array}$ & $\begin{array}{l}\text { Erro } \\
\text { padrão }\end{array}$ & $\beta$ & $\begin{array}{c}\beta \\
\text { padronizado }\end{array}$ & $\begin{array}{l}\text { Erro } \\
\text { padrão }\end{array}$ \\
\hline \multicolumn{10}{|l|}{$\operatorname{Sexo}^{*}$} \\
\hline Masculino & 19,9 & 0,15 & 4,1 & 59,9 & 0,25 & 7,9 & 68,3 & 0,27 & 6,2 \\
\hline Feminino & 9,6 & 0,13 & 1,9 & 35,1 & 0,27 & 3,9 & 40,1 & 0,35 & 3,2 \\
\hline \multicolumn{10}{|l|}{ Idade ${ }^{* *}$} \\
\hline 14-15 anos & -- & -- & -- & -- & -- & -- & 54,2 & 0,31 & 5,4 \\
\hline 16-19 anos & -- & -- & -- & -- & -- & -- & 43,6 & 0,30 & 4,1 \\
\hline \multicolumn{10}{|c|}{ Classe econômica*** } \\
\hline$A / B$ & -- & -- & -- & 38,2 & 0,19 & 5,8 & 53,1 & 0,33 & 4,9 \\
\hline$C / D / E$ & -- & -- & -- & 54,4 & 0,27 & 5,8 & 42,3 & 0,28 & 4,4 \\
\hline
\end{tabular}

*Ajustado por idade, classe econômica e índice de massa corporal. ${ }^{* *}$ Ajustado por sexo, classe econômica e índice de massa corporal. ${ }^{* * *}$ Ajustado por sexo, idade e índice de massa corporal. Todos os resultados apresentaram valor $p<0,001$. -- Não apresentou moderação $(p<0,05)$.

\section{Discussão}

Os resultados deste estudo demonstraram que a associação entre fatores psicossociais e o nível de atividade física dos adolescentes foi moderada pelos fatores sociodemográficos. As magnitudes das associações entre a autoeficácia, apoio social dos pais e dos amigos e a prática de atividade física foram mais elevadas nos adolescentes do sexo masculino. Também foram identificadas maiores magnitudes nas medidas de associação nos adolescentes de classe econômica média-baixa para o apoio social dos pais e nos de classe econômica alta e mais jovens para o apoio social dos amigos.

Estudos de revisão sistemática ${ }^{1,3}$ têm consistentemente identificado a percepção de autoeficácia e o fornecimento de apoio social de pais e dos amigos como importantes fatores psicossociais associados a maiores níveis de atividade física em adolescentes. No presente estudo, tanto a percepção de autoeficácia como o apoio social dos pais e amigos se associarou de forma positiva ao nível de atividade física dos adolescentes de ambos os sexos, porém com maior magnitude de asso- 
ciação naqueles do sexo masculino. Em geral, os adolescentes do sexo masculino são fisicamente mais ativos ${ }^{4}$, praticam mais esportes e atividades coletivas ${ }^{15}$ e são socialmente mais valorizados em relação à prática de atividade física, principalmente aquelas com maior exigência de esforço físico ${ }^{16}$. Tais fatores contribuem para que eles se percebam como sendo mais habilidosos, encorajados e com maior capacidade física para superar seus limites para a prática de atividade física ${ }^{11,17}$. Dentre estes, por exemplo, estariam a superação do sentimento de "preguiça", desmotivação e falta de tempo ${ }^{11,17}$.

O maior fornecimento de apoio social para os adolescentes do sexo masculino está frequentemente relacionado a maior liberdade por parte dos pais em permitirem que seus filhos encontrarem amigos fora do ambiente escolar, frequentem praças, parques e quadras esportivas ou simplesmente brinquem na rua, comparados aos do sexo feminino ${ }^{18}$. Já o apoio social fornecido pelos amigos está vinculado a partilha de valores, normas, gostos e preferências por prática de atividade física entre estes, aumentando o potencial de influência dos amigos sobre a atividade física nesse subgrupo ${ }^{19}$. O contrário disto é observado nos adolescentes do sexo feminino que, na maioria das vezes, relatam mais experiências negativas em atividades físicas como, por exemplo, machucados e falta de habilidade decorrente da pouca vivência em diferentes tipos de atividades físicas ${ }^{20}$; suas preferências por atividades mais sedentárias como assistir televisão, falar ao telefone ou "navegar na internet"20; priorizam atividades de natureza social como a demanda das atividades acadêmicas, tarefas domésticas ${ }^{18,20}$ e recebem maiores restrições e limites para sair de casa e encontrar com os amigos ${ }^{18}$.

Observou-se no presente estudo que a magnitude de associação entre apoio social dos amigos e prática de atividade física foi maior nos adolescentes mais novos (14-15 anos de idade) comparados aos mais velhos (16-19 anos de idade). Adolescentes mais jovens são fisicamente mais ativos e influenciados pelos amigos a seguirem os mesmos comportamentos, incluindo a prática de atividade física ${ }^{18}$. Já os adolescentes mais velhos preferem as atividades físicas individualizadas, são mais exigidos para se dedicarem aos estudos, cursos preparatórios, estágios e ao trabalho ${ }^{18}$. Esses fatores ajudam a diminuir a prioridade que é dada às atividades físicas ${ }^{18}$, reduzindo a possibilidade de exposição ao apoio social de outros grupos sociais. Desse modo, o apoio social dos amigos teria uma menor influência na prática de atividade física nos adolescentes mais velhos, por estarem menos expostos à influencia e o contato direto com os amigos.

O fornecimento de apoio social, a influência das normas sociais do grupo de convívio e interesses semelhantes por determinados comportamentos estão entre as principais formas de influência exercida pelos amigos, principalmente aqueles mais jovens $s^{3,18,21}$. Em estudo longitudinal desenvolvido com adolescentes escoceses, Kirby et al. ${ }^{22}$ identificaram que o apoio social dos amigos aumentava em até três vezes as chances de os adolescentes serem mais ativos fisicamente com o passar da adolescência, reforçando a ideia de que com o passar dos anos a influência social exercida pelos amigos tende a aumentar. É importante considerar também que a influência social dos amigos sobre a prática de atividade física dos adolescentes pode ser viabilizada por meio dos diferentes tipos de apoio social, como, o apoio em forma de práticas em conjunto e o fornecimento de comentários positivos em relação à prática ${ }^{23}$, assim como, em decorrência do possível papel modelador da prática dos amigos ${ }^{21}$.

No presente estudo, as associações entre o apoio social dos pais e dos amigos para a prática de atividade física apresentaram magnitudes distintas conforme a 
classe econômica em que os adolescentes se encontravam. De forma geral, os estudos têm identificado que adolescentes de nível socioeconômico mais elevado apresentam maiores níveis de prática comparados aqueles de menor nível ${ }^{3,24}$. As explicações para isso estão baseadas no fato de que essas famílias reconhecem mais a importância dos benefícios da atividade física para saúde dos filhos, possibilitam aos filhos que estudem em escolas com condições mais favoráveis à prática de atividades físicas (mais opções e melhor infraestrutura para a prática), matriculam os mesmos em clubes ou associações desportivas, têm maior capacidade para aquisição de materiais para as práticas e fornecem transporte para os locais de prática ${ }^{3,24}$.

Ao contrário do que era esperado, o apoio social dos pais se mostrou mais importante nos adolescentes de classe econômica média/baixa. As explicações para esse resultado podem estar relacionadas ao fato de que os pais de classe econômica média/baixa dão mais apoio para que os filhos realizem mais atividades físicas no contexto do deslocamentos (ir á pé para escola, casa de amigos ou vizinhança) e realizem atividades não estruturadas como jogos e brincadeiras ${ }^{16}$. Entre os adolescentes que pertencem as classes econômicas mais baixas essas atividades apresentam uma elevada contribuição para o escore geral de atividade física, e podem impactar de forma significativa em sua prática de atividade física semanal ${ }^{25}$. É possível considerar também que os tipos de apoio social fornecidos pelos pais e o contexto no qual a atividade física foi realizada, possam ser fatores que variem em decorrência da classe econômica dos adolescentes e assim possam influenciar para maiores práticas nesse subgrupo.

Em relação ao apoio social dos amigos e prática de atividade física dos adolescentes, o presente estudo identificou uma maior magnitude na medida de associação destas variáveis para aqueles de classe econômica alta. Frequentemente, os adolescentes de classe econômica alta apresentam uma maior prática de atividades físicas estruturadas e em locais específicos, como clubes e associações desportivas $^{24}$. Essas práticas, por si só, são mais favoráveis para aumentar os vínculos de amizade $^{24}$ e, por conseguinte, a disseminação de comportamentos mais fisicamente ativos entre os amigos ${ }^{26}$.

As principais limitações deste estudo incluem: o estudo ser de delineamento transversal, não permitindo estabelecer uma relação causal da associação entre fatores psicossociais e prática de atividade física dos adolescentes, apesar dos resultados das associações estarem no sentido hipotético esperado e identificados em estudos longitudinais ${ }^{11,22}$. Deve-se considerar que as associações encontradas podem ser bidirecionais, de modo que as maiores magnitudes da medida de associação identificadas em determinados subgrupos podem ser decorrentes do fato de que esses já eram mais ativos fisicamente. Outra limitação é a medida do apoio social dos pais ter sido analisada de maneira agrupada ao invés de considerar uma escala de apoio social para o pai e outra para a mãe, isto é importante, uma vez que a associação entre apoio social e atividade física pode variar conforme a fonte de apoio ${ }^{23,27}$.

Este estudo apresenta alguns pontos fortes: a amostra foi representativa de escolares do ensino médio e com poder estatístico adequado para testar as hipóteses; o questionário utilizado na coleta de dados foi previamente validado e aplicado por equipe treinada; houve baixo percentual de perdas e recusas; sem diferenças significativas entre os adolescentes incluídos e excluídos das análises, e foram considerados fatores de confusão importantes para esse tipo de estudo (sexo, idade, classe econômica e estado nutricional).

Conclui-se que a associação entre fatores psicossociais e a prática de atividade física em adolescentes é moderada por fatores sociodemográficos, sendo que para 
alguns subgrupos esses fatores se mostraram mais importantes do que outros. A maior percepção de autoeficácia e o maior fornecimento de apoio social dos pais e amigos foram mais importantes para os adolescentes do sexo masculino. $\mathrm{O}$ apoio social dos pais foi relevante entre os adolescentes de classe econômica média-baixa e o apoio social dos amigos foi importante entre os adolescentes de classe econômica alta e os mais jovens. Esses achados sugerem que apesar de a percepção de autoeficácia e o apoio social dos pais e amigos terem se mostrado associados a maiores níveis de atividade física, as suas possíveis influências na prática dos adolescentes variam de acordo com as características sociodemográficas dos mesmos. Intervenções para aumentar o nível de atividade física devem considerar esses achados ao planejarem e desenvolverem ações para aumentar a percepção de autoeficácia e o apoio social dos pais e amigos.

\section{Agradecimentos}

À Coordenação de Aperfeiçoamento de Pessoal de Nível Superior (CAPES) pela bolsa de estudos e pesquisa. À Secretaria de Educação da Paraíba, aos gestores, professores e alunos das escolas que participaram do estudo. Aos estagiários do Curso de Educação Física que fizeram parte da equipe de coleta de dados.

\section{Contribuições dos autores}

Cheng. L.A. participou da concepção do artigo, análise dos dados, revisão de literatura e redação do manuscrito. Mendonça, G. participou revisão de literatura e redação do manuscrito. Farias Júnior, J.C. participou de todas as etapas do estudo, incluindo concepção do artigo, coleta e análise dos dados e revisão crítica do manuscrito.

\section{Referências}

1. Sterdt E, Liersch S, Walter U. Correlates of physical activity of children and adolescents: a systematic review of reviews. Health Educ J. 2013;73(1):72-89.

2. Seabra AF, Mendonça DM, Thomis MA, Anjos LA, Maia JA. Biological and socio-cultural determinants of physical activity in adolescents. Cad Saude Publica. 2008;24(4):721-36.

3. Bauman AE, Reis RS, Sallis JF, Wells JC, Loos RJ, Martin BW. Correlates of physical activity: why are some people physically active and others not? Lancet. 2012; Series (380):258-71.

4. Hallal P, Andersen LB, Bull FC, Guthold R, Haskell W, Ekelund U. Global physical activity levels: surveillance progress, pitfalls, and prospects. Lancet. 2012; Series (380):247-57.

5. World Health Organization. Global recommendations on physical activity for health. World Health Organization. Geneva, Switzerland: World Health Organization; 2010.

6. Dumith SC, Gigante DP, Domingues MR, Kohl HW. Physical activity change during adolescence: a systematic review and a pooled analysis. Int J Epidemiol. 2011;40(3):685-98.

7. Azevedo MR, Horta BL, Gigante DP, Sibbritt D. Continuidade da atividade física na Coorte de Nascimentos de 1982 de Pelotas. Rev Bras Ativ Fis Saude. 2011;16(2):156-61.

8. Telama R, Yang X. Decline of physical activity from youth to young adulthood in Finland. Med Sci Sports Exerc. 2000;32(9):1617-22.

9. Van der Horst K, Paw MJCA, Twisk JWR, Mechelen WV. A brief review on correlates of physical activity and sedentariness in youth. Med Sci Sports Exerc. 2007;39(8):1241-50.

10. Wenthe PJ, Janz KF, Levy SM. Gender similarities and differences in factors associated with adolescent moderate-vigorous physical activity. Pediatr Exerc Sci. 2009;21(3):291-304.

11. Dishman RK, Saunders RP, Motl RW, Dowda M, Pate RR. Self-efficacy moderates the relation between declines in physical activity and perceived social support in high school girls. J Pediatr Psychol. 2009;34(4):441-51.

12. Farias Júnior JC, Lopes AS, Mota J, Santos MP, Ribeiro JC, Hallal PC. Validity and reproducibility of a physical activity questionnaire for adolescents: adapting the SelfAdministered Physical Activity Checklist. Rev Bras Epidemiol. 2012;15(1):198-210. 
13. Farias Júnior JC, Lopes AS, Reis RS, Nascimento JV, Borgatto AF, Hallal PC. Development and validation of a questionnaire measuring factors associated with physical activity in adolescents. Rev Bras Saúde Matern Infant. 2011;11(3):301-12.

14. Brazil Economic Classification Criterion - [database on the Internet] 2009 [cited November 26th, 2009]. Available from: http://www.abep.org/new/codigosCondutas.aspx.

15. Silva SM, Knuth AG, Duca GFD, Camargo MBJ, Cruz SH, Castagno V, et al. Individual and collective sports practice and associated factors among adolescents belonging to a birth cohort study. Rev Bras Educ Fis Esp. 2009;23(3):263-74.

16. Allender S, Cowburn G, Foster C. Understanding participation in sport and physical activity among children and adults: a review of qualitative studies. Health Educ Res. 2006;21(6):826-35.

17. Shields CA, Spink KS, Chad K, Muhajarine N, Humbert L, Odnokon P. Youth and adolescent physical activity lapsers: Examining self-efficacy as a mediator of the relationship between family social influence and physical activity. J Health Psychol. 2008;13(1):121-30.

18. Gonçalves H, Hallal PC, Amorim TC, Araújo CLP, Menezes A. Sociocultural factors and physical activity level in early adolescence. Rev Panam Salud Pública. 2007;22(4):246-53.

19. Okun MA, Ruehlman L, Karoly P, Lutz R, Fairholme C, Schaub R. Social support and social norms: do both contribute to predicting leisure-time exercise? Am J Health Behav. 2003;27(5):493-507.

20. Standiford A. The secret struggle of the active girl: a qualitative synthesis of interpersonal factors that influence physical activity in adolescent girls. Health Care Women Int. 2013:1-18.

21. Cheng LA, Mendonça G, Farias Júnior JC. Physical activity in adolescents: analysis of the social influence of parents and friends. J Pediatr (Rio J). 2014;90(1):35-41.

22. Kirby J, Levin KA, Inchley J. Parental and peer influences on physical activity among Scottish adolescents: a longitudinal study. J Phys Act Health. 2011;8(6):785.

23. Mendonça G, Farias Júnior JC. Physical activity and social support in adolescents: analysis of different types and sources of social support. J Sports Sci. 2015;33(18):1942-51.

24. Stalsberg R, Pedersen AV. Effects of socioeconomic status on the physical activity in adolescents: a systematic review of the evidence. Scand J Med Sci Spor. 2010;20(3):368-83.

25. Slingerland M, Borghouts LB, Hesselink MK. Physical activity energy expenditure in Dutch adolescents: contribution of active transport to school, physical education, and leisure time activities. J Sch Health. 2012 May;82(5):225-32.

26. Mendonça G, Cheng LA, Melo EN, Farias Junior JC. Physical activity and social support in adolescents: a systematic review. Health Educ Res. 2014;30(5):822-39.

27. Beets MW, Vogel R, Forlaw L, Pitetti KH, Cardinal BJ. Social support and youth physical activity: the role of provider and type. Am J Health Behav. 2006;30(3):278-89.

ENDEREÇO PARA

CORRESPONDÊNCIA

LUANNA ALEXANDRA CHENG

luanna_cheng@hotmail.com
Universidade Federal da Paraíba,

Departamento de Educação Física.

Campus I, Cidade Universitária.

CEP: $58059-900$ - João Pessoa, PB.

Fone: (83) 3216-7030.
RECEBIDO REVISADO APROVADO

$30 / 03 / 2016$ $08 / 06 / 2016$ 09/06/2016 